\title{
Perceived cardiovascular disease risk and tailored communication strategies among rural and urban community dwellers in Rwanda: A qualitative study
}

Jean Berchmans NIYIBIZI ( $\sim$ jeanberchmansniyibizi@yahoo.fr)

College of Medicine and Health Sciences (CMHS), University of Rwanda

Kufre Joseph OKOP

Chronic Diseases Initiative for Africa (CDIA), Department of Medicine, University of Cape Town Jean Pierre NGANABASHAKA

College of Medicine and Health Sciences (CMHS), University of Rwanda

Ghislaine UMWALI

College of Medicine and Health Sciences (CMHS), University of Rwanda

\section{Stephen RULISA}

College of Medicine and Health Sciences (CMHS), University of Rwanda

\section{Seleman NTAWUYIRUSHINTEGE}

College of Medicine and Health Sciences (CMHS), University of Rwanda

\section{David TUMUSIIME}

College of Medicine and Health Sciences (CMHS), University of Rwanda

\section{Alypio NYANDWI}

Ministry of Health

Evariste NTAGANDA

Rwanda Biomedical Center

\section{Peter Delobelle}

Chronic Diseases Initiative for Africa (CDIA), Department of Medicine, University of Cape Town Naomi LEVITT

Chronic Diseases Initiative for Africa (CDIA), Department of Medicine, University of Cape Town

Charlotte M. BAVUMA

College of Medicine and Health Sciences (CMHS), University of Rwanda

\section{Research Article}

Keywords: Cardiovascular disease, perceived risk, communication strategies, Rwanda

Posted Date: August 11th, 2021

DOI: https://doi.org/10.21203/rs.3.rs-799333/v1 
License: (c) (i) This work is licensed under a Creative Commons Attribution 4.0 International License. Read Full License 


\section{Abstract \\ Background}

In Rwanda, cardiovascular diseases (CVDs) are the third leading cause of death, and hence constitute an important public health issue. Like worldwide, most CVDs are due to lifestyle and preventable risk factors. Prevention interventions are based on risk factors for CVD risk, yet the outcome of such interventions might be limited by the lack of awareness or misconception of CVD risk. This study aimed to explore how rural and urban population groups in Rwanda perceive CVD risk and tailor communication strategies for estimated total cardiovascular risk.

\section{Methods}

An exploratory qualitative study design was applied using focus group discussions to collect data from rural and urban community dwellers. Thematic analysis with Atlas ti 7.5.18 was used and main findings for each theme reported as a narrative summary.

\section{Results}

Participants thought that CVD risk is due to either financial stress, psychosocial stress, substance abuse, noise pollution, unhealthy diets, diabetes or overworking. Participants did not understand CVD risk presented in quantitative format, but preferred qualitative formats or colors to represent low, moderate and high CVD risk through in-person communication. Participants preferred to be screened for CVD risk by community health workers using mobile health technology.

\section{Conclusion}

Rural and urban community members in Rwanda are aware of their CVD risk. Community health workers are preferred by local communities for CVD risk screening. Quantitative formats to present the total CVD risk appear inappropriate to the Rwandan population and qualitative formats are therefore advisable. Thus, operational research on the use of qualitative formats to communicate CVD risk is recommended to improve decision-making on CVD risk communication in the context of Rwanda.

\section{Background}

Cardiovascular disease (CVD) is one of the major public health issues worldwide. Globally, in 2017, 17.8 million people died of CVD[1]. Low- and middle- income countries (LMICs) were more affected as the rate of CVD death was 2.5 times higher than in high-income countries (HICs)[1].Before the 1990's, HICs accounted for the highest age standardized CVD mortality rate globally, but this has declined in the last 
decades due to the impact of preventive interventions[1].In LMICs, there is therefore a need to develop evidence-based and robust prevention strategies in order to stop or reverse the emerging CVD burden.

In 2013, the Word Health Organization (WHO)-STEPS survey on non-communicable diseases (NCDs) risk factors in Rwanda found that $19.1 \%$ of men and $7.1 \%$ of women were smokers, $30.0 \%$ of men and $17.0 \%$ of women were heavy drinkers (of alcohol) at the time of interview, and $99.1 \%$ of the population reported low fruit and vegetable intake[2]. Furthermore, CVD was the third leading cause of death, responsible for approximately $7.5 \%$ of total annual deaths in 2012[2].

The strategy of identifying and managing individuals at high risk of CVD is paramount for CVD prevention and control, in addition to other multi-sectoral population-based interventions targeting CVD risk factors in the entire population.[3] The prevention of CVD requires population awareness of the risk for developing CVD and risk reduction strategies[4].Awareness and understanding of personal risk is an important step for adopting healthy behaviors aimed at preventing ill-health, including CVD events $[5$, 7].This is in line with the constructs of the health belief model (HBM) as described by Jones et a[8], which state that an accurate evaluation of health risk is one of the critical factors of perceived personal vulnerability to such risk, which in turn informs health-seeking behavior. In the HBM model, selfevaluation of CVD risk is considered as a function of an individual's perception of vulnerability, understanding of the threat, the effect of societal motivations / demotivations and the perceived benefits of taking action to avert such risk[8].

The way in which information is conveyed plays an important role for understanding the level of CVD risk, which in turn determines health seeking behavior for risk prevention[9-10]. Graphic formats or bar charts and thermometer formats (with percentages) have, for example, been evaluated in previous qualitative studies conducted in Australia and their results are inconclusive to some degree[4,11]. This indicates the need for more contextualized research into CVD risk presentation formats, as results may differ from one setting to another. A specific need for research in CVD risk communication, including development of standardized tools that can be used by health workers, was indeed previously highlighted[4].

To the best of our knowledge no other study has been conducted in Rwanda in regard to CVD risk communication. This study was undertaken to explore the understanding of, and perception towards, CVD risk and CVD risk communication methods among rural and urban community dwellers to inform evidence-based policies and practices in Rwanda.

\section{Methods}

\section{Study design}

The study used an exploratory qualitative design to explore community members' understanding of selfperception and tailored communication strategies towards CVD risk among rural and urban dwellers. In this study, the term 'community' was operationalized as the catchment area of a health center (HC) providing NCDs (including CVD) services. 


\section{Setting, study population and sampling strategy}

One HC in Burera district, Northern Province and one HC in Gasabo district, City of Kigali were conveniently selected as rural and urban study sites, respectively. The study population included people with limited education (completed secondary education and below) and aged 18-65 years. Data were collected through focus group discussions (FGDs) with community members recruited from the local community. For the sake of homogeneity among participants, FGDs were divided by gender (men and women) and age group (young: 18-24 years old and adult: 25-65 years old) to enhance participants' comfort and relevance to their life stage. In total, four FGDs were conducted in each site (rural / urban), including two FGDs with youth (one with men and one with women), and two FGDs with adults (one with men and one with women).

Using a volunteer sampling strategy, ordinary community members were recruited within the catchment area of the selected HCs. They were selected using the existing forums for social cohesion at village level, including young people forums and parents' evening forums which are held monthly in each village. In doing so, one cell (the basic political-administrative unit composed by villages in Rwanda) was randomly selected in the catchment area of each selected $\mathrm{HC}$, followed by the selection of two villages within each cell. The first village served to recruit participants of FGDs with youth and the second village served to recruit participants of FGDs with adults.

One of the eligibility criteria for participating in FGDs was an adequate level of literacy and numeracy. Community members who voluntarily manifested interest to participate in the study, were tested against these criteria, and those who passed were retained.

\section{Data collection}

Data were collected using a FGD guide that was developed taking into consideration Health Belief Model (HBM) constructs. Participants were first asked general questions exploring common or well-known diseases in the local community and the knowledge about the heart and its function in the human body. They were asked questions about what they think can harm their heart; about how they perceive the possibility of being exposed to things that can affect their heart; and about how they can explain heart disease. FGD participants were also asked about their health seeking actions in the prior 12 months, make them believe that a disease is very harmful and the things that are in their local community that can cause general ill-health. They were also asked to cite what might cause harm or danger to their heart in daily life, and how information around the possibility or likelihood of having a disease or dying from disease had been communicated in their communities. They were also asked how they would want the message on having a heart related disease, or the likelihood of dying of a heart related disease to be communicated to them. The ability of FGD participants to understand their disease risk compared to others was assessed by asking them the following question: "'If a doctor tells you that the likelihood of you having a particular disease e.g. is five or more times than that of your friend or neighbour of the same age, how would you understand or interpret this statement from the Doctor." Lastly, FGD participants were given narrative and visual (thermometer and bar chart) formats indicating different 
levels of risk (risk score) and asked to interpret these (for more details, see FGD guide in Appendix 1). Each FGD was moderated by two researchers who were part of the investigators team and experienced in FGD facilitation; JBN served as the facilitator whereas JPN or GU served as notes taker. FGD lasted between $1 \mathrm{~h} 45 \mathrm{~min}$ and $2 \mathrm{~h} 46 \mathrm{~min}$ and data collection took place during the period September-November 2019.

\section{Analysis}

Data were analyzed using thematic analysis following the six steps described by Vaismoradi, et al.[12]: (i) familiarising with the data; (ii) generating initial codes; (iii) searching for themes; (iv) reviewing themes; (v) defining and naming themes; and (vi) producing the report. Even though the FGD topic guide was conceived using HBM constructs, an inductive approach was used during analysis in order to allow the data to determine themes. The analysis was performed using Atlas.ti 7.5.18.

Prior to analysis, eight FGD recordings were transcribed verbatim in the local language (Kinyarwanda) and translated into English. Analysis was done by three researchers (JBN, GU \& JPN), who all read the first FGD transcript of the rural study site. To ensure inter-rater consistency, these researchers met to compare individual codes and resolve discrepancies. All three researchers continued independent analysis using the second transcript of the FGD conducted in the urban study site. Newly identified codes and themes were discussed in another meeting. Using the codes agreed upon during this meeting, one researcher / analyst (JBN) continued analysis of the six remaining FGDs. JBN then shared the updated thematic codebook with GU and JPN for discussion until consensus was reached. Afterwards, JBN continued with the phases of reviewing themes, defining and naming themes, and producing the final analysis report.

Results were reported as a narrative summary of main findings for each theme and one or two selected quotes from FGD participants.

\section{Findings}

Overall, 77 FGD participants were recruited, but $12(16 \%)$ did not attend their allocated FGDs. The median age (in years) of study participants was 29 (22-39), a little more than half were women $(n=34 ; 52.3 \%)$, and more than half were adult $(n=39 ; 60 \%)$.

The findings are presented in the form of themes that emerged from the FGDs and are pertaining to CVD risk communication. These themes and their sub-themes are provided in Table 1 below: 
Table 1

Themes and sub-themes of FGD transcript analysis

\begin{tabular}{|lll|}
\hline Number & Themes & Sub-themes \\
\hline 1 & $\begin{array}{l}\text { Perception on } \\
\text { disease related } \\
\text { harm }\end{array}$ & $\begin{array}{l}\text { Chronicity of a disease, being selective for foods and drinks, } \\
\text { diseases leading to sudden death, impediment to physiological } \\
\text { functions, fatality if no medicine taken, disease symptoms and } \\
\text { length of hospital stay }\end{array}$ \\
\hline 3 & $\begin{array}{l}\text { Perceived risk of } \\
\text { CVD }\end{array}$ & $\begin{array}{l}\text { Financial stress, psychosocial stress, substance abuse, noise } \\
\text { pollution, unhealthy diet, diabetes and overworking }\end{array}$ \\
\hline 4 & $\begin{array}{l}\text { Free and voluntary } \\
\text { screening for CVD } \\
\text { risk factors }\end{array}$ & $\begin{array}{l}\text { Existing ways of } \\
\text { communicating } \\
\text { disease or death } \\
\text { risk }\end{array}$ \\
\hline 5 & $\begin{array}{l}\text { Outreach campaign, lack of communication of a disease or risk of } \\
\text { dying at health facility level }\end{array}$ \\
\hline $\begin{array}{l}\text { Preferences of CVD } \\
\text { risk screening and } \\
\text { communication }\end{array}$ & $\begin{array}{l}\text { Using mobile health technology for CVD (risk) screening, CVD risk } \\
\text { screening by community health workers (CHWs), preferred ways of } \\
\text { communicating the likelihood of a disease or death }\end{array}$ \\
\hline $\begin{array}{l}\text { Perception towards } \\
\text { counseling services } \\
\text { at health facility }\end{array}$ & $\begin{array}{l}\text { Inadequate counseling services at health facility, unawareness of } \\
\text { existing NCD (CVD) services at health facility }\end{array}$ \\
\hline 7 & $\begin{array}{l}\text { Interpretation or } \\
\text { understanding of } \\
\text { the (CVD) risk } \\
\text { presentation } \\
\text { formats }\end{array}$ \\
\hline
\end{tabular}

\section{Perception on disease related harm}

Across all FGDs, chronicity of a disease, being selective to foods and drinks, diseases leading to sudden death, disease that impedes some physiological functions, attack rate, fatality if no medicine taken, disease symptoms and length of hospital stay are factors which were perceived as being linked to a harmful disease.

During this study, we assessed which color represents a high risk by giving each participant a piece of paper with four different colors (red, yellow, green and white), as indicated in Fig. 1, and asking them to identify one color that represents a high risk. Red color was perceived by almost all study participants to be related to the highest risk.

With the same piece of paper, FGD participants were also asked to choose one colour they think can be used to represent the least (or smallest) possible harm. Across all FGDs, participants reported that either orange or green colours may be used to represent the lowest risk. However, some participants mentioned that white can also be used to represent the least (or smallest) possible harm. 


\section{Perceived risk of CVD}

When participants were asked about their perceived risk of suffering from CVD, those who perceived to be at risk attributed this to either financial stress, psychosocial stress, substance abuse, noise pollution, unhealthy diet, diabetes, or overworking.

\section{Financial stress}

Across FGDs, participants expressed that they are likely to suffer from CVD because of financial constraint. Financial constraint was perceived as either failure to sustain a small business, incapacity to feed their family, bankruptcy, or poverty in general.

"Most of the people do not have income generating activities and those who try to venture into small business, they are not successful. In this life condition, people think much especially how to pay school fees for children or foodstuff which leads to living a misery life and consequently get attacked by various diseases including heart attack.": Participant \#2, FGD \#4 (Adult women), Urban site

"Poverty causes headache and heart disease." (Participant \#2, FGD \#2 (Adult women), Rural site

\section{Psychosocial stress}

In both study sites, FDG participants perceived themselves to be at CVD risk due to psychosocial stress. This was expressed as, e.g., deception in romantic relationships among young people, gender-based violence within couples, violence committed to children (heavily beaten by their parents), individual reactions towards a stressful situation (when they are told of or witness tragic events, they are told off etc.), being gossiped, experiencing (or being told about) a tragic event, anxiety, overthinking daily life and depression.

"It's what they were talking about the husband; constant worries and stress caused by your husband at the point you feel like you are about to lose your mind. Another thing is a tragic event when you lose your relative, it might cause you harm or danger to your heart.": Participant \#9, FGD \#2 (Adult women), Rural site

"Another thing, in our region parents like to beat their children, so you may find a child is always afraid and traumatized which can lead him/her to have a heart disease.":

Participant \# 1 (Young men), Urban site

\section{Substance abuse}

Young men from both communities, and adult men from the urban community perceived themselves to be at risk from CVD because of the consumption of alcoholic beverages. Most participants who reported alcohol consumption also smoked cigarettes and perceived themselves to be at risk of CVD. Only adult 
and young men who self-reported smoking cigarettes or illicit drugs (like marijuana) which put them at CVD risk.

"For me, three things can cause troubles to my heart. First of all, I smoke cigarette. Secondly, I drink beer and thirdly, I think so much such that my heart never get rest.": Participant \#5, FGD \#3 (Adult men), Urban site

One adult woman from the urban community perceived herself to be at risk of suffering from CVDs because of secondhand smoke produced by marijuana smoked by young people in their local community.

"For me, drugs can also cause trouble to our hearts especially for youth and generally for our children. When it comes to the evening, young people lock themselves and smoke marijuana, and smell is widespread to non-smokers and affect them without leaving the smokers behind.": Participant \#4, FGD \#4 (Adult women), Urban site

\section{Noise pollution}

Across FGDs, some participants claimed to be exposed to environmental noise which may put them at risk of CVD. The sources of environmental noise were bars, churches, loud music played on the radio or factories found in their neighborhood (like cassava flour making machines).

"Thank you. We are living with young people who play loud music in the mid night. I think that this noise can cause troubles to our heart in the future..." Participant \#3, FGD \#4 (Adult women), Urban site

\section{Unhealthy diet}

In both study sites, FGD participants perceived that they are likely to suffer from CVD because of unhealthy diets. Unhealthy diet was expressed as eating either industrial / processed food, meat, too much oil or too much salt. One adult woman in the FGD conducted in the urban community attributed their habit of consuming unhealthy food to poverty, hindering them to afford a healthy diet.

"I also think that I may get a heart attack in the near future because of industrial food that contain toxics. Eating cow's meat or oil may also cause heart-related diseases. Healthy cooking oil are not affordable to poor people.": Participant \#11, FGD \#4 (Adult women), Urban site

In the urban community, women (both young and adult) revealed that they eat food based on deliciousness and not on their nutritious function.

"... We are also eating food based on deliciousness not on the nutritious importance. We seem to ignore what are the negative consequences that may occur in the future. Certainly, I may get heart attack in the future.": Participant \#3, FGD \#4 (Adult women), Urban site

\section{Diabetes}


One young man in the urban site asserted that he perceived herself to be at CVD risk because he was diagnosed with diabetes risk.

"Last year I went to the hospital and the doctor told me to stop taking much sugar because I was at risk of having diabetes, and diabetes can lead to heart diseases.": Participant \#4, FGD \#7 (Young men), Urban site

\section{Overworking}

Adult study participants from the urban community reported that they may suffer from CVD because of overworking in order to satisfy their needs.

"For instance, I went to hospital last week and after testing me they told me that I have to take enough rest in order to allow my heart function well. But I find it is difficult to rest because I have to work tirelessly in order to get money and satisfy my needs.": Participant \#4, FGD \#3 (Adult men), Urban site

\section{Free and Voluntary Screening for CVD risk factors}

Only one participant in the FGD with men from the urban community reported to be aware of the car free day promoting mass sports in which the Rwanda Ministry of Health and Development partners avail medical staff and kits to screen car free day participants for CVD risk factors like hypertension and diabetes for free.

" ...there is a chance to get tested these NCDs for free of charge during the "Sports for All" an event organized by the $\mathrm{MoH}$ that is held once a month. Some people feared to go to hospital due to lack of financial means but now it is high time to participate in that "sports for all" so as to have a chance to get tested and know their health status.": Participant, FGD \#3 (Adult men), Urban site

Only in the FGD with adult women in the urban community, two participants revealed that they voluntarily go to seek CVD risk factor screening services from their health facility. One of these participants reported to be surprised for being diagnosed with a CVD risk factor (hypertension):

"What I can tell you is that even last week I was sick. When I went to hospital for medical check-up, the test results showed that I have blood pressure which is obviously related to heart disease. I could not imagine that I can have such blood pressure and it was high blood pressure...": Participant \#2, FGD \#4 (Adult women), Urban site

Although some FGD participants were aware of the opportunity of accessing free CVD (risk) screening, other FGD participants were not aware of this opportunity:

"From what I know, not everyone has been mobilized to regularly go for medical check-up so to know his/her health status in order to take the preventive measures. It is possible that someone can spend more than five years without going to the hospital to know his / her health status and that is why some community members die abruptly because of disease they did not know before. People do not have the 
same level of understanding where one can advise herself to go for medical check-up.": Participant \#9, FGD \#4 (Adult women), Urban site

\section{Existing ways of communicating disease or death risk}

In both study sites, we found that disease awareness in the community is conducted in the form of meetings, whereby health professionals provide information about disease outbreaks (e.g. increase of malaria cases) or a new disease for which community members are at risk (e.g. Ebola). In these outreach campaigns, health professionals teach the community about symptoms, causes, mode of transmission, prevention of the concerned disease, and sometimes screen participants for that disease and treat positive cases.

"If there is an emerging disease where health care providers will notice a sudden increase of patients suffering from that disease, like malaria for example. In that case, the health centre organizes an outreach program where they come in the community and teach how to prevent that disease and test us on site for that disease.": Participant, FGD \#1 (Adult men), Rural site

Communication of the likelihood of getting or dying from a disease is almost non-existent. Across all FGDs, only one participant asserted that a doctor can tell a person that he has the likelihood of getting a disease:

"...the doctor may tell you that they diagnosed diabetes as an example; or they tell you that you are about to get this disease, and maybe the medicines they give you, should reduce the severity; or they just put you on a diet.": Participant \#6, FGD \#6 (Young women), Rural site

Only one participant in the FGD with adult women from the rural community shared a story about her friend who had been told by a doctor about the likelihood of her mother dying:

"I once had a conversation with someone who had a patient about dying and he told me the following: the patient had two daughters who were looking after her, she was about to die and she asked for water. The doctor after seeing that young daughter is so demoralized, he took her to another room to talk to her. He told her the situation about her mother and prepared her to what was about to happen. Right after coming back from where she was having a conversation with the doctor, her mother had already died. She wasn't surprised by the death of her mother as the doctor prepared her for that. Counseling is important.": Participant, FGD \#2 (Adult women), Rural site

In the rural community, participants in the FGD with adult women reported that doctors / health care providers do not openly communicate about the likelihood of dying of a disease, but their facial expressions may help caregivers to guess that their patients are dying soon:

"Except when a patient is about to die.... You may just bring a person and take care of him/her, there is quite a difference for someone who is about to die and for someone who isn't. Sometimes you may find that a doctor / health care provider is demoralized to treat him / her [a patient] and sometimes he / she 
tells you to take back the patient at home, sometimes you can observe in the face of the doctor / health care provider and you say that I am working hard in vain. S/he cannot show you that he / she [patient] is dying while s/he has not yet died....»: Participant \#9, FGD \#2 (Adult women), Rural site

\section{Preferences on CVD Risk Screening and Communication}

FGD participants suggested using technology in screening for CVD risk, for example by using (smart) phones. In the urban community, it was suggested that community health workers be trained on the use of mobile health (m-Health) technology to conduct door-to-door CVD (risk) screening and provide medications:

"I have an idea, it would be better if community health workers have that system and pass to every family, for example if they have that machine that check and give immediate results and medicaments.": Participant, FGD \#7 (Young men), Urban site

Across FGDs, most participants preferred to be told about the likelihood of a disease in the form of (private) conversation or counseling:

"I wish my doctor / health care provider to give me a message in a way of counseling. I would like him / her to talk to me privately and advise me what to do, how to behave but not everyone to know my case.": Participant, FGD \#3 (Adult men), Urban site

"...S/he can start the discussion with simple things, and continue telling you about that disease, how can someone contract it, what can cause it and its symptoms. He can finish the discussion by telling you that you have the likelihood of having that disease in the future.": Participant, FGD \#8 (Adult men), Rural site

Only young men from the rural community FGD suggested to present the likelihood of a disease happening to a person using colours. Some of them suggested to use the red colour to present high risk; yellow the moderate risk and green the absence of or low risk:

"....For example if you use a red colour, you explain that it represents someone at the highest level, a yellow colour represents someone who can be treated and cured; green colour someone who is safe.": Participant \#6, FGD \#5 (Young men), Rural site

\section{Perception towards counseling services at the health facility}

FGD participants reported not being provided with adequate counseling services at the health facility. They reported that there are some health services providers who poorly communicate with patients. This was indicated by saying e.g., that health providers do not share information about their disease after conducting diagnostic tests; when they talk to them they do so in unfriendly ways, as per the quotes below: 
"... My wife and I go to the hospital more often but whenever we go there, we explain to the health care provider the way we feel and the latter give only medicine without telling us what we are suffering from.": Participant \#2, FGD \#1 (Adult men), Rural site

"I think that s/he can talk to you in a good way advising you to stop anything bad to your health because there are some who talk so badly with pressure and stressing you which is not good, but when s/he talks to you kindly you can follow what you are ordered and have a good follow up.": Participant \#5, FGD \#8 (Young women), Urban site

In the rural community, study participants suggested having one health provider specialized or trained in CVDs who can provide CVD services. This expressed need can be perceived as an indicator that community members are not aware of, or sensitized on NCDs services provided at the $\mathrm{HC}$ as the selection of study sites was based on the criteria of a community ( $\mathrm{HC}$ catchment area) served by a $\mathrm{HC}$ providing NCDs services and having a nurse in charge of NCDs (including CVD) services.

" Health centers don't have doctors / health services providers who treat heart disease, it could be better if we have one at the health center specialized only on that.": Participant, FGD \#1 (Adult men), Rural site

\section{Interpretation or understanding of (CVD) risk presentation}

In the context of testing the ability of community members to interpret disease risk presented in the form of either a thermometer or a bar graph whereby each FGD participant was given a piece of paper displaying samples of risk presentation, and asked: "If the health services provider gives you your medical test results in the form of that piece of paper, does it help you to appreciate or evaluate your likelihood of having a disease?" most participants provided irrelevant answers. Findings from each risk presentation are described below:

Thermometer without label on the arrow indicating the level of likelihood of having a disease in the next five years (Fig. 2): only one participant from the FGD with young men in the urban community provided the right answer.

Thermometer with label on the arrow indicating the level of likelihood of having a disease in the next five years (Fig. 3): across all FGDs no participant was able to guess the message conveyed by the risk presentation format.

Bar graphs with a label under each (rectangular) bar (Fig. 4a\&4b): only a few participants from the FGD with adult men in the rural community and the FGD with young women in the urban community were able to guess the message conveyed by this risk presentation format.

Overall, we found that community members are not able to interpret / understand disease risk, neither when presented in thermometer format nor in bar graph format. FGD participants indicated that they do not understand these formats because of their low level of education. Some suggested explaining or 
communicating disease risk verbally (in person communication) instead of giving them a piece of paper and requesting them to guess the interpretation.

"This method is not understandable 100\% because not everyone can read it and get the meaning of his results, according to the capacity of the country not everyone has education, some have it, others don't. Not every person can explain him/herself that, so that's why I say that not everyone can know his / her results of the problem he has.": Participant \#1, FGD \#7 (Young men), Urban site

When assessing the ability of FGD participants to understand their disease risk compared to others, they were asked the following question: "If a doctor tells you that the likelihood of you having a particular disease e.g. is five or more times than that of your friend or neighbour of the same age, how would you understand or interpret this statement from the Doctor?" FGD participants did not understand the question and some FGD participants were only able to correctly interpret / answer the question after it was rephrased by the researcher.

"If he tells me that I have five times chance of having particular disease more than my friend, I would think that I'm about to get seriously sick.": Participant \#8, FGD \#1(Adult men), Rural site

"I think that, one is more exposed to the risk of getting that disease and get impacts than me.": Participant \#5, FGD \#7 (Young men), Urban site

Despite repeated efforts made by the researcher to rephrase and contextualize the question, some FGD participants were unable to understand the sentence, and some participants declared that they didn't understand the sentence at all, gave incorrect or vague or strange answers, and confused disease risk with the actual disease, as illustrated in the quotes below:

"If the doctor gives me an example that my brother's vulnerability is five times than mine. I can take the signs my brother has and multiply them by $5 \%$ in order to know the degree of my vulnerability. From there, I can draw a conclusion that based on that \%, I am going to respect doctor's advice in order to reduce the degree of vulnerability.": Participant \#1, FGD \#4(Adult women), Urban site

“We do not really understand.": Participant \#7, FGD \#6 (Young women), Rural site

One participant from the urban FGD with young women provided an interpretation opposing the real message conveyed by the statement:

"I think that s/he is trying to tell me that my friend is very sick.": Participant, FGD \#8 (Young women), Urban site

When asked about alternative ways to present disease risk, FGD participants in the rural community suggested using colours like yellow, green and red in order to represent different levels of risk:

"My suggestion is that on the drawing you used, there can be another way of making them more understandable; e.g. the colors you used: yellow, green and red, you could have written something like a 
label on each of the color indicating what they mean. That's my suggestion.": Participant \#2, FGD \#1 (Adult men), Rural site

\section{Discussion}

This study aimed at providing health services stakeholders and decision makers with information on the understanding of, and perception towards CVD risk and CVD risk communication among rural and urban community members with low educational attainment in Rwanda. It was found that study participants perceived themselves to be at risk of CVD because of financial stress, psychosocial stress, alcohol consumption, substance abuse, noise pollution, unhealthy diet, diabetes and overworking. Although there are opportunities of CVD risk factor screening free of charge, through for example car free day mass sports, study participants were not aware of this. This study also revealed that counseling services at health facilities were judged to be poor.

Importantly, this study revealed that CVD risk communication services do not exist in health facilities that were part of our study site. It was found that study participants have inadequate health literacy to understand and interpret CVD risk as presented in a thermometer or bar graph format and preferred receiving CVD risk communication through in-person or verbal communication. As regards CVD risk screening, study participants also preferred training ofCHWs to screen for CVD risk and using mobile phone technology. The culture of seeking CVD preventive healthcare services among study participants was quasi-inexistent, which is not in line with another study conducted in developed country, United States of America, where individuals attended health facilities for CVD preventive health care[13].

Factors perceived by study participants to lead to CVD risk were not different from findings of studies previously conducted in other contexts, including African countries. Stress which was perceived by most study participants to be a risk factor for CVD, was also reported by study participants in Kenya[14], Nigeria [15], South Africa[9] and America[16]. Moreover, stress was cited as the most perceived cause of heart disease in a recent qualitative meta-synthesis on CVD[17]. Stress in this study was linked to financial constraint to afford basic needs, similar to a report of perceptions of heart-healthy behaviour among African American adults[16]. Stress was also linked to psychosocial stress related to gender based violence, which was also reported in a qualitative study conducted in Kenya[14]. The finding that some participants from this study, and particularly women from the rural community, perceived themselves to be at risk of CVDs because of witnessing or being told about tragic news or being told off, is in line with another study conducted in South Africa where respondents thought that hearing tragic news can cause strokes. [9]

Importantly, the finding that young people in this study perceived themselves to be at CVD risk seems not surprising as cases of hemorrhagic stroke have already been reported among young adolescents in Rwanda[18]. This indicates a need for population-based interventions targeting CVD prevention in all age groups. 
Poverty was indirectly perceived as a source of financial stress, which is in line with the findings from another study conducted in Kenya where poverty was considered as an underlying risk factor for CVD[14]. In addition, in this study, poverty was linked to unhealthy diets which also led study participants to feel at risk of CVDs, in line with a study conducted by Surka et a[[9], in which respondents associated poverty with unhealthy diets and felt at risk of CVDs because of this. In Nigeria, adolescents and young adults perceived that unhealthy (poor) diet could put them at CVD risk[15]. Unhealthy diet in this study was linked to eating industrial / processed food, meat, too much oil or too much salt. These findings are in line with findings from other studies that investigated healthy individuals' self-perception towards CVD risk in Kenya[9] and South Africa [14]. Unhealthy food were also attributed to poverty, whereby some community members would consume unhealthy food out of convenience, findings that are in agreement with another study conducted in America[16]. Some participants in the FGD with women reported to consume unhealthy food because of its deliciousness, which is in line with findings from a study recently conducted in another African context where women reported difficulties to avoid preparing fatty food because of the pressure to satisfy the tastes of their husbands[14]. This indicates that, in some African contexts, cultural beliefs can be perceived as contributing factors to CVD risk exposure.

CVD risk in this study was also linked to excessive or harmful alcohol consumption and tobacco. Some study participants who felt at risk of CVD due to alcohol consumption advanced stress as a pretext to engage in this unhealthy behaviour, an argument also recorded in a study conducted in Kenya[14]. Other participants in this study did not acknowledge to be at CVD risk due to substance abuse because of lack of knowledge, which was also found in a study conducted in Kenya, where it was reported that most study participants were not aware of the association between smoking and excessive harmful alcohol consumption and CVD[14].

Environmental pollution, in the form of flour particulates produced by cassava flour making machines, was reported in this study as a risk factor for CVDs in the urban community, which was also found in another study on CVD risk factors in urban informal settings in Kenya[14]. In the latter study, dwellers reported that kerosene, wood charcoal for cooking, gaseous emissions and effluents from industries expose them to health risk[14].

Lack of awareness around opportunities to screen for CVD risk factors through mass sports, such as car free day sport, could be perceived as a proxy indicator of ineffective sensitization around CVD prevention which, in turn, could be translated into weak primary CVD prevention. Study participants in this study were not aware of CVD screening and treatment services at their nearest health facilities (health centers), which is in line with a study which reported the need to improve the quality of primary prevention for CVD in LMICs[19]. The suggestion / preference of study participants to train CHWs on CVD screening and to conduct door-to-door CVD screening were also recorded in another study in the region, where study participants suggested that community health volunteers can be used to conduct CVD screening in the community[14]. Moreover, in this study, FGD participants suggested using mobile health technology to screen individuals for CVD risk. This concurs with the study in Kenya where short messaging service and the use of social media platforms were suggested as strategies to disseminate messages on CVD risk, 
available opportunities to screen, and advice on where care and treatment could be sought[14]. One of the supporting ideas for involving CHWs in CVD primary prevention is the awareness of their success in other areas of health services provision, which was also found in the study in Kenya [14].

Being able to understand risk is only one of the factors that influence how people understand and use information in their decision-making [9]. Unfortunately, in this study, participants were unable to understand and interpret different CVD risk presentation formats, including thermometer and bar graph formats indicating the probability of a CVD event within five years. This inability to understand and interpret CVD risk was also noticed in a study recently conducted in South Africa[9], whereas both thermometer and bar graph formats were understood and preferred among patients and general practitioners in Australia[11]. The inability to understand and interpret CVD risk was also found in another study where the presentation of CVD risk using quantitative risk formats was only understood by highly educated individuals[20].

In this study, in-person conversation or counseling was suggested as a means to communicate CVD risk, which was also in line with findings from the study conducted in Australia, where qualitative formats were preferred over quantitative formats[20]. In the present study, participants reported to not understand quantitative formats because of their low level of education, which is not surprising as many people find it difficult to comprehend basic probabilities[10]. However, individual characteristics such as age, education and numeracy, may also affect risk comprehension and its qualitative interpretation[21].

A major strength of this study is that it considered both urban and rural areas, and youth and adults. The study was limited to community members with a low level of education and does not reflect the risk perception of individuals with higher levels of education (i.e., who attended at least university or any other tertiary level of education). However, it was reasonable to conduct this study among individuals with a low level of education since the proportion of individuals who attended university in Rwanda is 1.9\%[22].

In our opinion, this qualitative study provides useful information to policy makers and programme implementers in designing programmes to enhance awareness on CVD risk and risk factors and insights into how to deliver effective CVD prevention and care, particularly in Rwanda and in low-resourced communities in general.

\section{Conclusion}

This study showed that some rural and urban community members, in Rwanda, are aware of their risk of CVD; however, others may not be aware of their risk because of their lack of knowledge of CVD (risk factors). This study also showed that quantitative formats to present CVD risk are less suitable within the context of Rwanda. As a result, we recommend using qualitative formats (low risk, moderate risk, and high risk) to present CVD risk. If quantitative formats are used, this should be accompanied by qualitative interpretation (and counselling sessions) from health professionals. Community based-CVD risk screening conducted by CHWs are likely to be accepted by local community dwellers. The use of colours could also be a possible way to represent CVD risk. In our opinion, this qualitative study provided useful 
information to policy makers and programme implementers in designing programmes to enhance awareness on CVD risk and risk factors and insights into how to deliver effective CVD prevention and care, particularly in Rwanda and in low-resourced communities in general. Action research about the capacities of CHWs to screen their fellow community members for CVD risk using mobile heath technology is hence recommended. Operational research on the use of qualitative formats to communicate CVD risk is recommended to improve decision-making on CVD risk communication in the context of Rwanda as well.

\section{Abbreviations}

CHWs: Community health workers, CVD: cardiovascular disease, HBM: Health belief model, HC: Health centre, HICs: High-income countries, FGDs: Focus Group Discussions, LMICs: Low- and middle- income countries, MoH: Ministry of Health, NCDs: Non-communicable diseases, RNEC: Rwanda National Ethics Committee, STEPS: STEPwise approach to surveillance, WHO: Word Health Organization

\section{Declarations}

\section{Ethics approval and consent to participate}

The study protocol conforms to the ethical guidelines of the 1975 Declaration of Helsinki as reflected in a priori approval by the Institutional Review Board of the Rwanda Ministry of Health (MoH), the Rwanda National Ethics Committee (RNEC) (No.256/RNEC/2019). All study participants signed a written consent form before participating in the FGD.

\section{Consent for publication}

This is not applicable as individual person's details, images or video are not being presented.

\section{Availability of data and materials}

The data for this study are available from the corresponding author (JBN) upon reasonable request.

\section{Competing interests}

The authors declare that they have no competing interests

\section{Funding}

This work was funded by the German Federal Ministry of Education and Research (BMBF) as part of the Research Networks for Health Innovations in sub-Saharan Africa Funding Initiative through the Collaboration for Evidence-based Healthcare and Public Health in Africa (CEBHA+) project (Number: 
66.3010.7-002.09). The funding agency did not take part in any step of this study from study design to the submission of the manuscript.

\section{Authors' contributions}

JBN was the lead author, contributed to the study methodology, data collection and analysis, and drafted the initial manuscript. JKO contributed to study conceptualization and methodology, and revised the manuscript for scientific insight. JPN and GU contributed to data collection and analysis, and revised the manuscript. NL,SR, SN, DT, EN and AN contributed to study conceptualization, methodology, and review of the manuscript. PD contributed to the review of the manuscript. BCM contributed to the study conceptualization, methodology, research implementation, initial manuscript drafting and provided insight in the revised manuscript. All authors gave their final approval for the version to be published.

\section{Acknowledgements}

Not applicable.

\section{References}

1 Jagannathan R, Patel SA, Ali MK, and Narayan KMV, "Global Updates on Cardiovascular Disease Mortality Trends and Attribution of Traditional Risk Factors," Curr. Diab. Rep., vol. 19, no. 7, pp. 1-12, 2019, doi: 10.1007/s11892-019-1161-2.

2 Rwanda Ministry of Health, "Rwanda Non-communicable Diseases Risk Factors Report," https://www.who.int/ncds/surveillance/steps/Rwanda_2012_STEPS_Report.pdf [Accessed on December 12,2020], Kigali, 2015.

3 Bovet P, Chiolero A, Paccaud F, and Banatvala N, "Screening for cardiovascular disease risk and subsequent management in low and middle income countries: Challenges and opportunities," Public Health Rev., vol. 36, no. 1, pp. 1-15, 2015, doi: 10.1186/s40985-015-0013-0.

4 Webster R and Heeley E, "Perceptions of risk: Understanding cardiovascular disease," Risk Manag. Healthc. Policy, vol. 3, pp. 49-60, 2010, doi: 10.2147/RMHP.S8288.

5 Grauman, Hansson M, James S, Veldwijk J, and Höglund A, “Exploring research participants' perceptions of cardiovascular risk information-Room for improvement and empowerment," Patient Educ. Couns., vol. 102, no. 8, pp. 1528-1534, 2019, doi: 10.1016/j.pec.2019.03.010.

6 Ko Y and Boo S, "Self-perceived health versus actual cardiovascular disease risks," Japan J. Nurs. Sci., vol. 13, no. 1, pp. 65-74, 2016, doi: 10.1111/jjns.12087. 
7 Bonner $\mathrm{C}$ et al., "Should heart age calculators be used alongside absolute cardiovascular disease risk assessment?," BMC Cardiovasc. Disord., vol. 18, no. 1, pp. 1-8, 2018, doi: 10.1186/s12872-018-07601.

8 Jones WJ, Jensen JD, Scherr CL, Brown NR, Christy K, "The Health Belief Model as an Explanatory Framework in Communication Research: Exploring Parallel, Serial, and Moderated Mediation," Heal. Commun, vol. 6, no. 30, pp. 566-576, 2015, doi: 10.1080/10410236.2013.873363.

9 Surka S, Steyn K, Everett-Murphy K, Gaziano TA, and Levitt N, "Knowledge and perceptions of risk for cardiovascular disease: Findings of a qualitative investigation from a low-income peri-urban community in the Western Cape, South Africa," African J. Prim. Heal. Care Fam. Med., vol. 7, no. 1, pp. 18, 2015, doi: 10.4102/phcfm.v7i1.891.

10 Navar AN, Stone N, "What to Say and How to Say It: Effective Communication for Cardiovascular Disease Prevention," Curr Opin Cardiol, vol. 5, no. 31, pp. 537-544, 2016, doi:

10.1097/HCO.0000000000000322.

11 Hill S et al., "Absolute risk representation in cardiovascular disease prevention: Comprehension and preferences of health care consumers and general practitioners involved in a focus group study," $B M C$ Public Health, vol. 10:108, pp. 1-13, 2010, doi: 10.1186/1471-2458-10-108.

12 Vaismoradi $\mathrm{M}$, Turunen $\mathrm{H}$, and Bondas $\mathrm{T}$, "Content analysis and thematic analysis: Implications for conducting a qualitative descriptive study," Nurs. Heal. Sci., vol. 15, no. 3, pp. 398-405, 2013, doi: $10.1111 /$ nhs. 12048 .

13 Mosca $L$ et al., "National study of women's awareness, preventive action, and barriers to cardiovascular health," Circulation, vol. 113, no. 4, pp. 525-534, 2006, doi:

10.1161/CIRCULATIONAHA.105.588103.

14 Wekesah FM, Kyobutungi C, Grobbee DE, and Klipstein-Grobusch K, "Understanding of and perceptions towards cardiovascular diseases and their risk factors: A qualitative study among residents of urban informal settings in Nairobi," BMJ Open, vol. 9, no. 6, 2019, doi: 10.1136/bmjopen-2018-026852.

15 Odunaiya NA, Adesanya T, Okoye EC, and Oguntibeju 00, "Towards cardiovascular disease prevention in Nigeria: A mixed method study of how adolescents and young adults in a university setting perceive cardiovascular disease and risk factors," African J. Prim. Heal. care Fam. Med., vol. 13, no. 1, pp. 1-9, 2021, doi: 10.4102/phcfm.v13i1.2200.

16 Der Ananian C, Winham DM, Thompson SV, and Tisue ME, "Perceptions of heart-healthy behaviors among African American adults: A mixed methods study," Int. J. Environ. Res. Public Health, vol. 15, no. 11, 2018, doi: 10.3390/ijerph15112433. 
17 Tulu SN, N. Al Salmi, and Jones J, "Understanding cardiovascular disease in day-to-day living for African people: a qualitative metasynthesis," BMC Public Health, vol. 21, no. 1, pp. 1-14, 2021, doi: 10.1186/s12889-021-10781-1.

18 Mushumba $\mathrm{H}$ et al., "The burden of stroke in young black population and the role of post mortem evaluation in increasing awareness and prevention," Rwanda Med. J., vol. 72, no. 4, pp. 26-28, 2015.

19 Gupta R and Yusuf S, "Challenges in management and prevention of ischemic heart disease in low socioeconomic status people in LLMICs," BMC Med., vol. 17, no. 1, pp. 1-11, 2019, doi: 10.1186/s12916019-1454-y.

20 Bonner $\mathrm{C}$ et al., "Communicating cardiovascular disease risk: An interview study of General Practitioners' use of absolute risk within tailored communication strategies," BMC Fam. Pract., vol. 15, no. 1, pp. 1-8, 2014, doi: 10.1186/1471-2296-15-106.

21 Navar AM et al., "Influence of Cardiovascular Risk Communication Tools and Presentation Formats on Patient Perceptions and Preferences," JAMA Cardiol., vol. 3, no. 12, pp. 1192-1199, 2018, doi:

10.1001/jamacardio.2018.3680.

22 National Institute of Statistics of Rwanda and Rwandan Ministry of Finance and Economic Planning, "Rwanda Fourth Population and Housing Census. Thematic Report: Educational characteristics of the population," Kigali, 2014. [Online]. Available: http://microdata.statistics.gov.rw/index.php/catalog/65/related_materials.

\section{Figures}

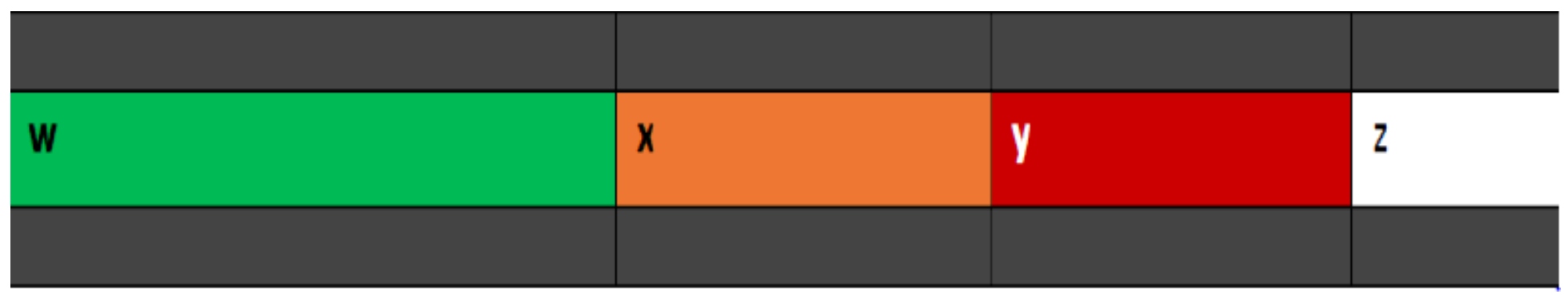

Figure 1

Colours that may be used to represent risk

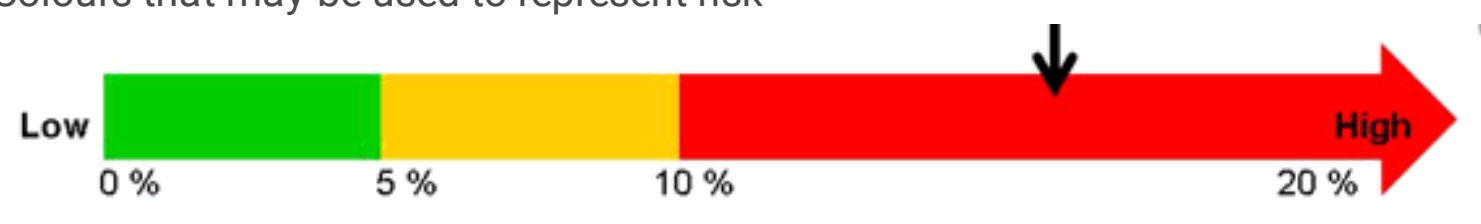

Figure 2 
Risk shown in a thermometer without explanation text

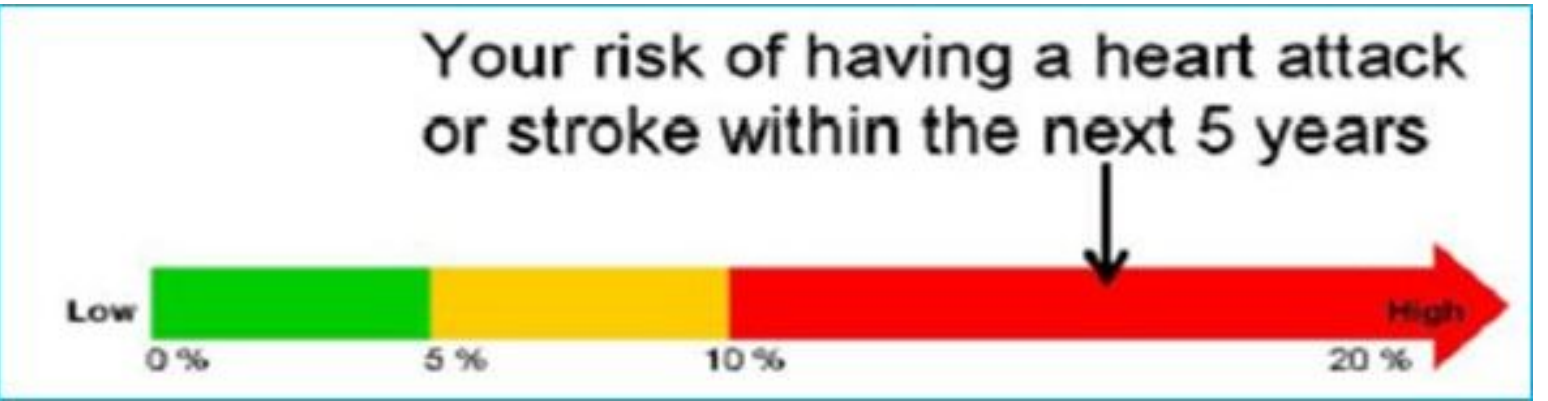

Figure 3

Risk shown in a thermometer with explanation text 
Figure 4a: CVD risk presented in bar graph format

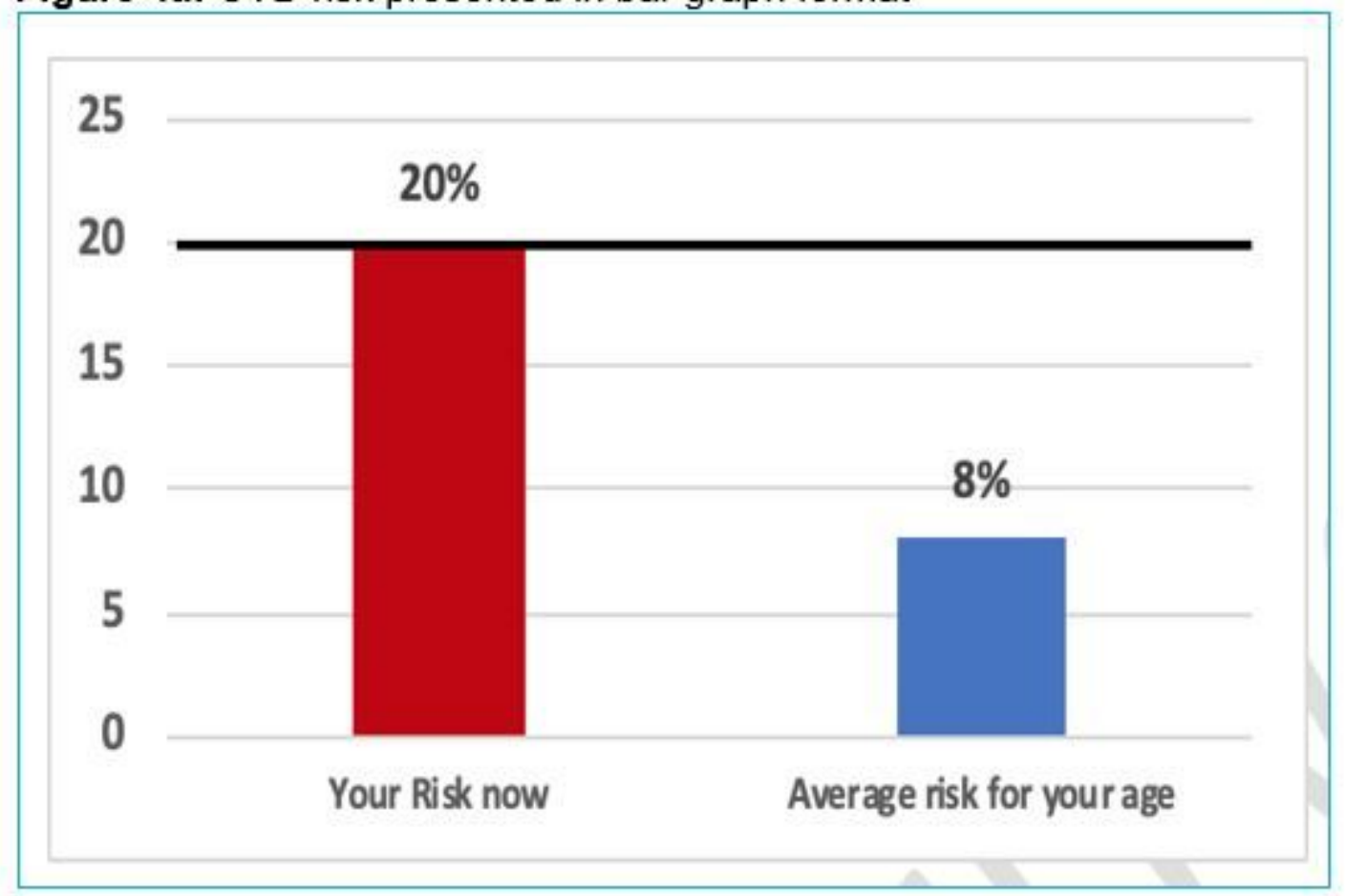

Figure 4b: CVD risk presented in bar graph format

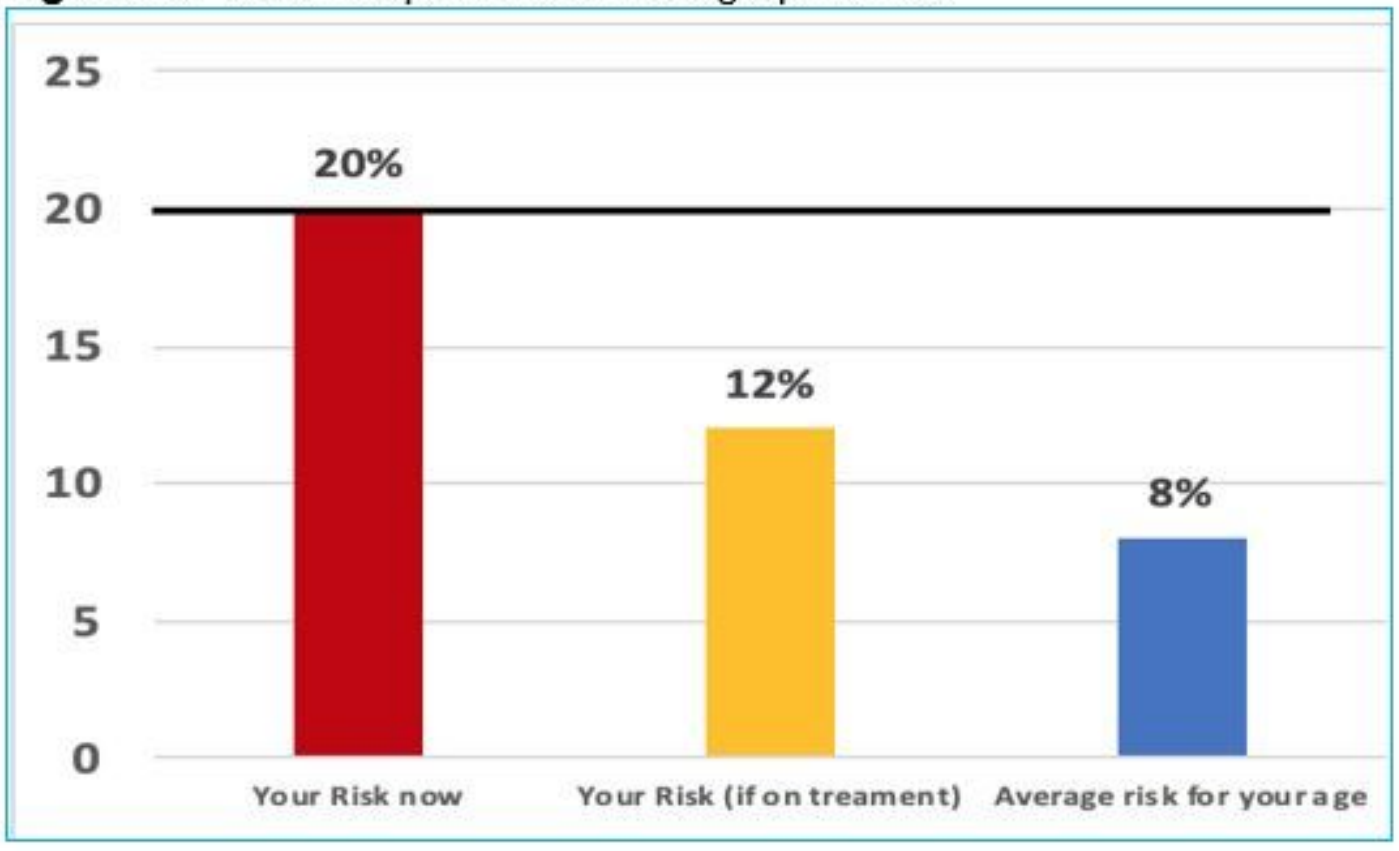

Figure 4

Figure 4a: CVD risk presented in bar graph format. Figure 4b: CVD risk presented in bar graph format.

\section{Supplementary Files}

This is a list of supplementary files associated with this preprint. Click to download. 
- AppendixFGDGuide.docx.pdf

Page 24/24 